\title{
CMR for Assessment of Diastolic Function
}

\author{
Jos J. M. Westenberg
}

Published online: 29 January 2011

(C) The Author(s) 2011. This article is published with open access at Springerlink.com

\begin{abstract}
Prevalence of heart failure with preserved left ventricular ejection fraction amounts to $50 \%$ of all cases with heart failure. Diagnosis assessment requires evidence of left ventricular diastolic dysfunction. Currently, echocardiography is the method of choice for diastolic function testing in clinical practice. Various applications are in use and recommended criteria are followed for classifying the severity of dysfunction. Cardiovascular magnetic resonance (CMR) offers a variety of alternative applications for evaluation of diastolic function, some superior to echocardiography in accuracy and reproducibility, some being complementary. In this article, the role of the available CMR applications for diastolic function testing in clinical practice and research is reviewed and compared to echocardiography.
\end{abstract}

Keywords Magnetic resonance imaging · Diastolic function - Left ventricle - Mitral valve · Velocity-encoding · Tagging $\cdot$ Pulmonary venous flow Three-dimensional . Image processing . Flow $\cdot$ Myocardial wall motion $\cdot$ Strain

\section{Introduction}

Diastole is the time period in the cardiac cycle between closure of the aortic valve (i.e., the onset of isovolumic relaxation) and closure of the mitral valve after the filling of the left ventricle (LV) has taken place. During diastole, relaxation of the ventricles occurs and due to a build-up

J. J. M. Westenberg $(\bowtie)$

Department of Radiology, Leiden University Medical Center, Albinusdreef 2,

2333 ZA, Leiden, The Netherlands

e-mail: j.j.m.westenberg@lumc.nl pressure difference between ventricles and atria, blood will flow through the atria-ventricular valves (i.e., mitral valve and tricuspid valve) into the ventricles. Atrial contraction will contribute to the final part of the ventricular filling process, after which the atria-ventricular valves will close. Diastole is an active, ATP-consuming myocardial process which is influenced by many factors, such as LV and atrial geometry and systolic function, performance of LV relaxation and atrial contraction, loading conditions, viscoelastic properties, pericardial restraint, valve function, heart rate, and the cardiac conduction system. The final combined effect of all these factors determines the interplay of the transmitral pressure gradient and the filling properties, which determines the LV filling.

Up to $50 \%$ of the patients that present with congestive heart failure (HF) reveal a normal or near normal LV ejection fraction (EF) $[1,2]$. Patients with symptomatic diastolic dysfunction will be classified with diastolic HF or, in case of isolated diastolic dysfunction, with HF with normal or preserved EF. Evidence of systolic dysfunction may be present in patients with diastolic HF [3], whereas diastolic dysfunction often but not exclusively occurs in systolic HF patients [4].

Diastolic dysfunction can be classified into four classes (Table 1). In the first phase of diastolic dysfunction, classified as grade I, the proportion of LV filling in the early phase of diastole is reduced. As diastolic functioning worsens, pressure in the left atrium increases resulting in a recovery of LV filling during the early phase, representing a pseudo-normal situation. The filling occurs under nonnormal physiological conditions, though, with the blood being actively forced into the LV by the increased pressure in the atrium, instead of being sucked into the LV due to the transmitral pressure gradient. This pseudo-normal situation is classified as grade II dysfunction. The next phase 
Table 1 Classification of diastolic dysfunction

\begin{tabular}{ll}
\hline Class diastolic dysfuntion & Description \\
\hline Grade I & Reduced filling in the early phase \\
Grade II & Pseudo-normal filling by increased atrial pressure \\
Grade III & Reversible restrictive filling due to impaired relaxation \\
Grade IV & Irreversible restrictive filling \\
\hline
\end{tabular}

represents restrictive filling due to impaired relaxation with elevated filling pressures and impaired LV compliance. This impaired filling may be reversible when the Valsava maneuver is performed (grade III). Grade IV, the worst phase of diastolic dysfunction, represents irreversible restrictive filling.

According to the consensus statement by the Heart Failure and Echocardiographic Associations of the European Society of Cardiology [5], diagnosis of HF with preserved LV EF must meet the following three criteria: 1) presence of symptoms or signs of HF; 2) presence of normal or near normal systolic function; and 3) evidence of diastolic dysfunction. Evaluation of LV diastolic function is paramount to distinguish diastolic dysfunction from other disease with similar symptoms, in order to determine optimal treatment and prognosis for the patient. Imaging is indispensable for diagnosis assessment in clinical practice. Invasive assessment of the LV pressure-volume relation, pulmonary capillary wedge pressure, and mean left atrial pressure using micro manometer conductance catheters is considered the most accurate approach for diastolic function assessment [6], but the risk of complications during this procedure is significant. In clinical practice, diastolic function is usually evaluated from echocardiography in combination with color Doppler, tissue Doppler imaging, and strain analysis from speckle-tracking. Committees of the American Society of Echocardiography and European Association of Echocardiography have published guidelines and recommended criteria for the evaluation of LV diastolic function by echocardiography in clinical practice and in research [7••]. In this article, the available applications of cardiovascular MR (CMR) for diastolic function testing as an alternative to echocardiography are reviewed.

\section{Time-Volume Relation and Left Atrial Size}

Two-dimensional and three-dimensional echocardiography is used in clinical practice for LV volume and function estimation but accuracy and reproducibility are limited. CMR is considered the gold standard for assessment of LV volumes and systolic function since this modality achieves superior image quality, especially over two-dimensional echocardiography, high reproducibility and sufficient spa- tial and temporal resolution, has minimal operatorvariability, and there are no limitations regarding choice of imaging volume $[8 \bullet, 9]$. The time-volume relation of the LV cavity is usually obtained from a series of breath-held time-resolved parallel short-axis planes (Fig. 1) or a radial stack of long-axis planes. Segmentation of the endocardial border and summation-of-discs reveal end-diastolic and end-systolic LV volumes, and the difference between both results in the stroke volume, which represents global systolic function. Manual segmentation can be very tedious and time-consuming as cine short-axis datasets usually amount to 300-500 images. Therefore, automated contourdetection methods are needed to facilitate analysis in clinical practice, but the performance of available algorithms varies with image quality. With the application of steady-state free-precession (SSFP) gradient-echo scan techniques at $1.5 \mathrm{~T}$, the image quality of cine LV shortaxis images increased dramatically compared to conventional gradient-echo sequences, resulting in a significant improvement of the delineation of the endocardial border [10]. Currently, off-resonance artifacts still limit the use of SSFP applications at higher field strength.

In the past, the LV time-volume relation was quantitatively used for evaluation of LV filling during diastole [1113]. In particular, early peak filling rate, time to peak filling rate, and atrial filling fraction were determined from the time-volume curve. This method is rarely used anymore since it does not provide information on filling characteristics and therefore, the use of time-volume relation for $\mathrm{LV}$ diastolic function assessment is limited.

Left atrial size is used as a classifier for diastolic dysfunction [7••, 14], as a significant relation between atrial remodeling and diastolic function indices exists. A value of left atrial volume indexed to body surface area exceeding $34 \mathrm{~mL} / \mathrm{m}^{2}$ has been shown to be an independent predictor of HF [15]. With echocardiography, atrial size is estimated from an apical 2-chamber and 4-chamber view using biplane Simpson. With MRI, a similar approach can be used to evaluate atrial remodeling (Fig. 2) [16, 17]. Alternatively, atrial size can be determined from cine shortaxis data that are acquired beyond the basal level of the heart [18], covering the atria as well as the ventricles. It is important to note that increased atrial size may be seen in $\mathrm{HF}$ in absence of diastolic dysfunction. Likewise, atrial dilatation often occurs in athletes. Therefore, measurement 

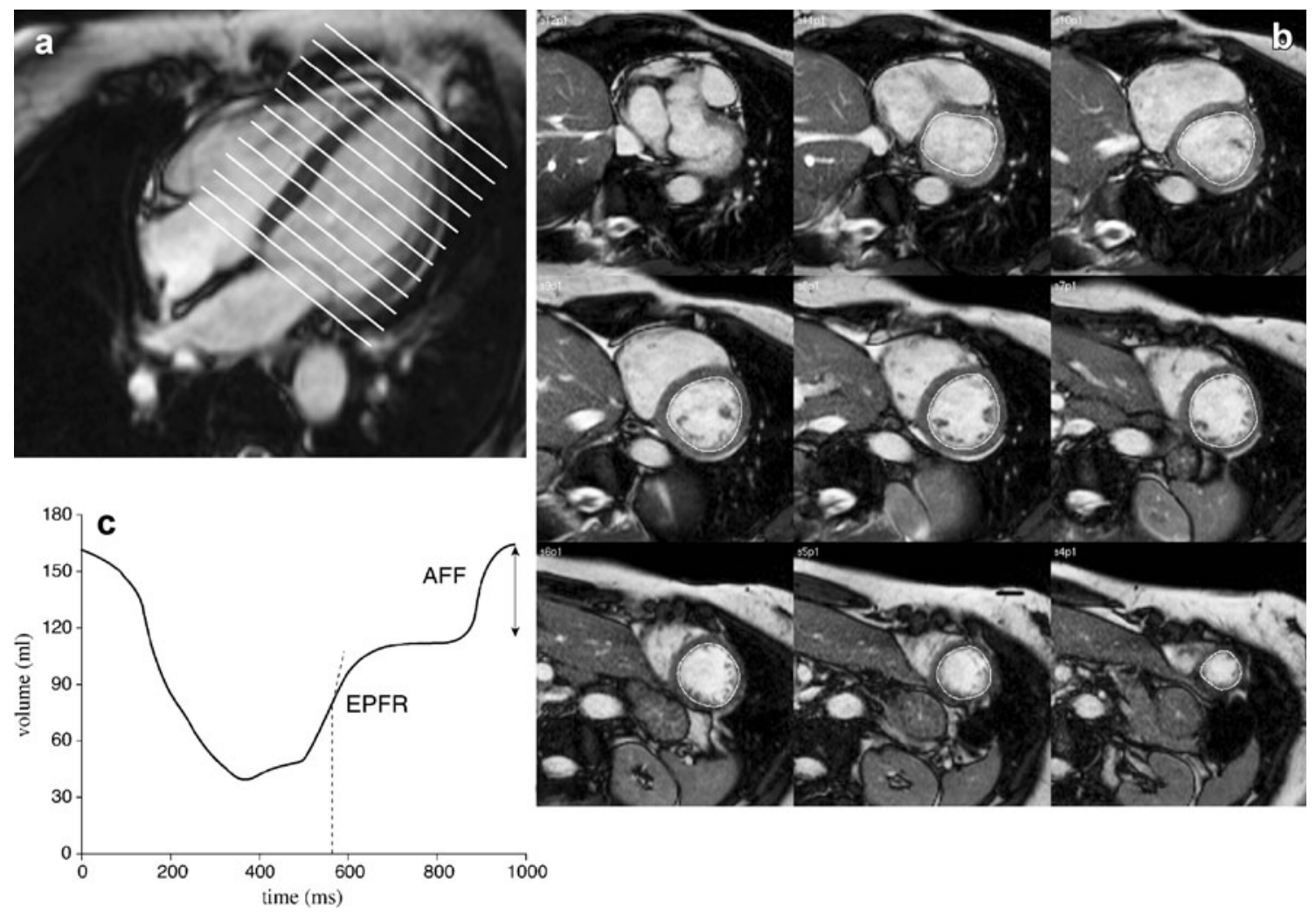

Fig. 1 LV time-volume relation (c) determined by planimetry from a multislice cine short-axis dataset (b), planned perpendicular to the long-axis of the LV (a). From the time-volume relation, early peak

filling rate (EPFR) is determined from the steepest gradient in the volume curve in the early filling phase. Atrial filling fraction (AFF) is determined in the atrial filling phase

of left atrial size should be only considered in combination with other indices of LV diastolic function when used as a predictor for HF.

\section{Mitral Inflow Pattern}

Assessment of mitral inflow velocities with pulsed-wave Doppler in combination with color Doppler in the apical four-chamber view [19] is one of the first simple tests performed when evaluating diastolic function. A $1-3 \mathrm{~mm}$

sample volume is positioned on the mitral leaflet tips during diastole to record the time-velocity curve. Wave form analysis results in quantification of early (E) and atrial (A) peak filling velocity, E/A ratio, and the deceleration time of the E peak filling velocity (DT), for which cutoff criteria are defined for diastolic dysfunction classification based on normal values $[7 \cdot \bullet]$. Normal diastolic function shows E/A between 0.8 and 1.5 and $\mathrm{DT}>200 \mathrm{~ms}$. In grade I dysfunction, E/A decreases below 0.8. In grade II, E/A moves back into the normal range of $0.8-1.5$, but DT decreases to values between 160 and $200 \mathrm{~ms}$. In grade III

Fig. 2 Left atrial size is determined by biplane Simpson's rule on the atrial areas determined in four-chamber (a) and two-chamber (b) view
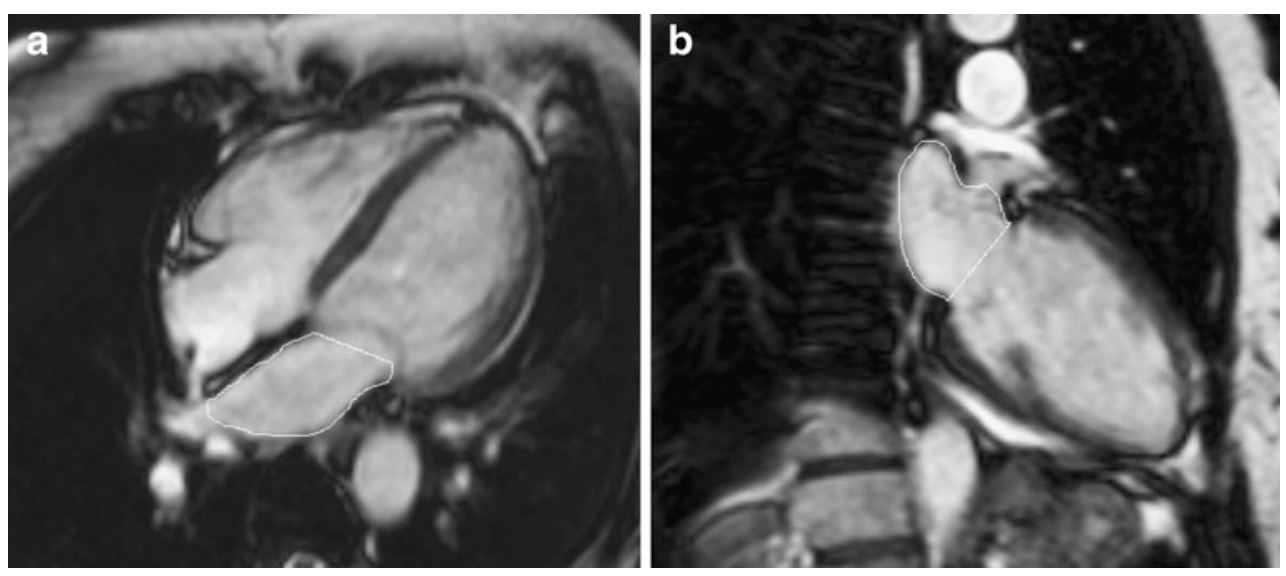
and IV, E/A increase to a value above 2 and DT drops below $160 \mathrm{~ms}$.

Velocity-encoded or phase-contrast MRI offers an attractive alternative for echo Doppler for quantifying intracardiac blood flow [11, 20-23]. Conventionally, a two-dimensional acquisition plane is placed at the position of the mitral valve at the moment of end-systole (Fig. 3). A time-resolved acquisition with velocity-encoding perpendicular to this plane (i.e., one-directional through-plane velocity-encoding) results in a time-velocity curve representing one average cardiac cycle. Thirty to forty cardiac phases are reconstructed resulting in a typical effective temporal resolution of the time-velocity curve of 20-30 ms. Similar to echo Doppler, wave form analysis of these timevelocity curves provides assessment of simple diastolic function indices such as E/A ratio and DT.

More often, the time-flow rate curve (i.e., transmitral flow rate) is used for wave form analysis since flow rate is less noise-sensitive than maximal velocity. For each cardiac phase, the mean velocity over the area of the annulus is determined. Contour definition is usually manually performed. It is recommended to perform through-plane motion correction in order to obtain the true transvalvular flow [24]. Although good correlation between echo Doppler studies and MRI has been reported, the cutoff values used in echocardiography cannot simply be translated to these MRI-assessed indices based on the time-flow rate curves. Furthermore, in this MRI approach similar as for echo Doppler, the acquisition plane is not adapted to the cyclic motion of the mitral annulus during contraction and relaxation but remains fixed during acquisition at the location of the mitral valve at the moment of end systole. Therefore, velocity during diastole will be acquired mainly inside the left ventricle below the mitral valve. Furthermore, the imaging plane will not be adapted to possible changes in orientation of flow direction, which may result in a systematic underestimation of inflow velocity. Recently, acquisition and analysis techniques have been introduced which perform tracking of the basal level of the heart during acquisition [25] or retrospectively during image analysis, when velocity data is acquired in all three directions inside a three-dimensional volume containing the blood flow velocity field inside the myocardium [26•]. When quantifying net transmitral inflow and mitral regurgitation, these techniques have proven to be more accurate over the conventional approach of two-dimensional onedirectional through-plane velocity-encoded MRI with a fixed acquisition plane. Finally, three-directional threedimensional velocity-encoded MRI with retrospective valve tracking also showed better agreement with echo Doppler when discriminating restrictive filling patterns from other patterns $[27 \cdot \bullet]$.

\section{Isovolumic Relaxation Time}

After closure of the aortic valve at end systole, a fast pressure drop in the LV is established by active relaxation of the ventricle in an untwisting motion with a closed LV volume. This pressure gradient is responsible for the suction force during LV filling. When relaxation is impaired, LV pressure falls slowly, prolonging the time required for LV pressure to drop below atrial pressure, and therefore, mitral valve opening is delayed. The isovolumic relaxation time (IVRT) is defined as the time period between closure of the aortic valve and opening of the mitral valve. In grade I diastolic dysfunction, IVRT extends to a value over $100 \mathrm{~ms}$ while for restrictive LV filling patterns (grade III and IV), IVRT drops below $60 \mathrm{~ms}$. With echocardiography, IVRT is easily obtained from pulsedwave Doppler with a sample volume placed first in the LV outflow tract, registering the start of isovolumic relaxation at the moment of aortic valve closure, and registering the moment of mitral valve opening by sampling at the leaflets of the mitral valve. Echo Doppler is sampled with high temporal resolution resulting in a highly accurate definition of IVRT.

CMR in combination with repeated one-directional through-plane velocity-encoding at the LV outflow tract and at the mitral valve, or by applying the three-directional three-dimensional velocity-encoding approach with an acquisition volume capturing both the mitral valve as well
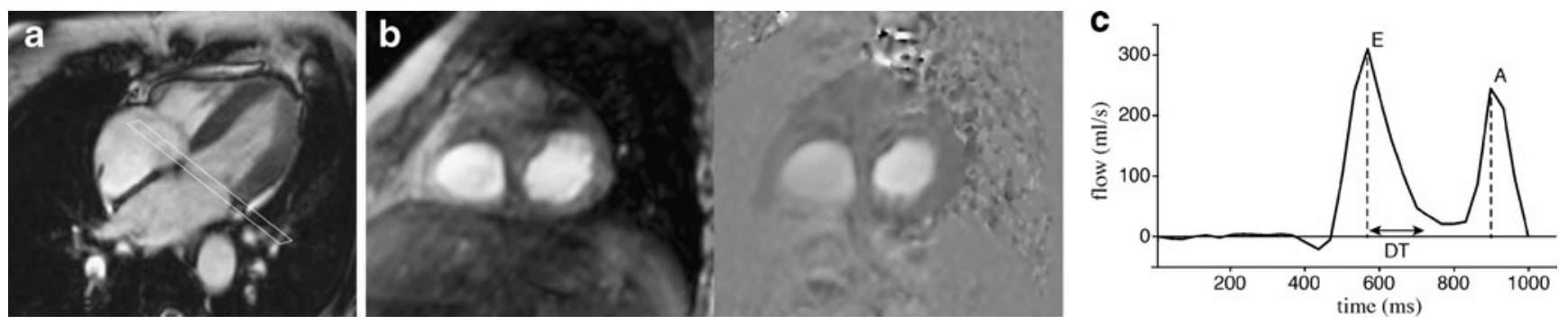

Fig. 3 At the moment of end-systole, an acquisition plane is positioned at the mitral valve (a). Through-plane one-directional velocity-encoded MRI (magnitude and velocity image in b) results in a time-flow rate graph (c), which is used for wave form analysis. Early (E) and atrial (A) peak filling rate can be determined, as well as the deceleration time (DT) of the E-peak 
as the aortic valve (Fig. 4) [28], should be able to register the moments of aortic valve closure and mitral valve opening. Drawbacks of this CMR application, however, are the inferior temporal resolution of velocity-encoded MRI compared to echo Doppler and the long MRI acquisition time. Alternatively, breath-held high temporal resolution SSFP cine imaging [29•] can be achieved with use of highend MRI scanners in combination with a 32-channel receive system and application of acceleration techniques based on spatial coil sensitivity variations (sensitivityencoding SENSE [30]) and spatiotemporal correlations (k-t broad-use linear-acquisition speedup technique, k-t BLAST [31]). Cine imaging with temporal resolution of $6 \mathrm{~ms}$ can be achieved, establishing improved accuracy for assessment of diastolic function indices such as E/A ratio, DT, and the definition of IVRT from time-volume curves and the first derivative of these curves [29•]. Also, the freedom in choosing any arbitrary imaging plane with CMR easily allows direct evaluation of valve functioning from a single cine acquisition of a three-chamber view which contains both the aortic valve as well as the mitral valve. The IVRT can be accurately assessed from this single breath-held cine acquisition in which the moments of valve closure and opening can be visually determined.

\section{Pulmonary Venous Flow}

LV filling and compliance, left atrial preload, and contractility all influence the filling pattern of the left atrium through the pulmonary veins, and therefore, wave form analysis of pulmonary venous flow velocity is a valuable tool in the evaluation of LV diastolic function. A pulsedwave Doppler acquisition of the pulmonary venous flow is performed in apical four-chamber view. The sample volume is typically placed $0.5 \mathrm{~cm}$ or further into the right upper pulmonary vein $[7 \bullet \bullet$. Wave form analysis of the pulmonary venous time-velocity curve includes assessment of peak systolic velocity (S), peak anterograde diastolic velocity (D), and peak atrial reversal velocity (Ar) in late diastole. From peak detection, indices as S/D-ratio, systolic filling fraction $\left(\mathrm{S}_{\text {time-velocity integral }} /\left[\mathrm{S}_{\text {time-velocity integral }}+\mathrm{D}_{\text {time-velocity integral }}\right]\right)$, and difference in duration of $\mathrm{Ar}$ and $\mathrm{A}$ peak from transmitral flow analysis are deducted. Usually, there are two systolic velocity peaks ( $\mathrm{S} 1$ and $\mathrm{S} 2$ ), mostly noticeable in case of an extended PR interval [32]. S1 is related to atrial relaxation, and S2 is related to atrial stroke volume and pulse wave propagation in the pulmonary artery. Therefore, S2 should be identified and used to determine $\mathrm{S} / \mathrm{D}$ ratio as an index of diastolic function. Normal values of pulmonary venous flow velocity indices are influenced by age $[7 \cdot \bullet]$, and S/D ratio will also increase with age, but changes in LV filling and compliance further affect the D velocity [33]. Also, Ar velocity will increase with age but this usually will not exceed $35 \mathrm{~cm} / \mathrm{s}$. Ar velocity and duration are both affected by late diastolic pressure in the LV, atrial preload, and contractility [34]. Therefore, values of Ar velocity exceeding $35 \mathrm{~cm} / \mathrm{s}$ suggest increased LV end-diastolic pressure [35]. The difference in duration between $\mathrm{Ar}$ and A peaks is the only age-independent indication of increased A wave pressure, which may be helpful for detection of elevated LV end-diastolic pressure (i.e., the first hemodynamic
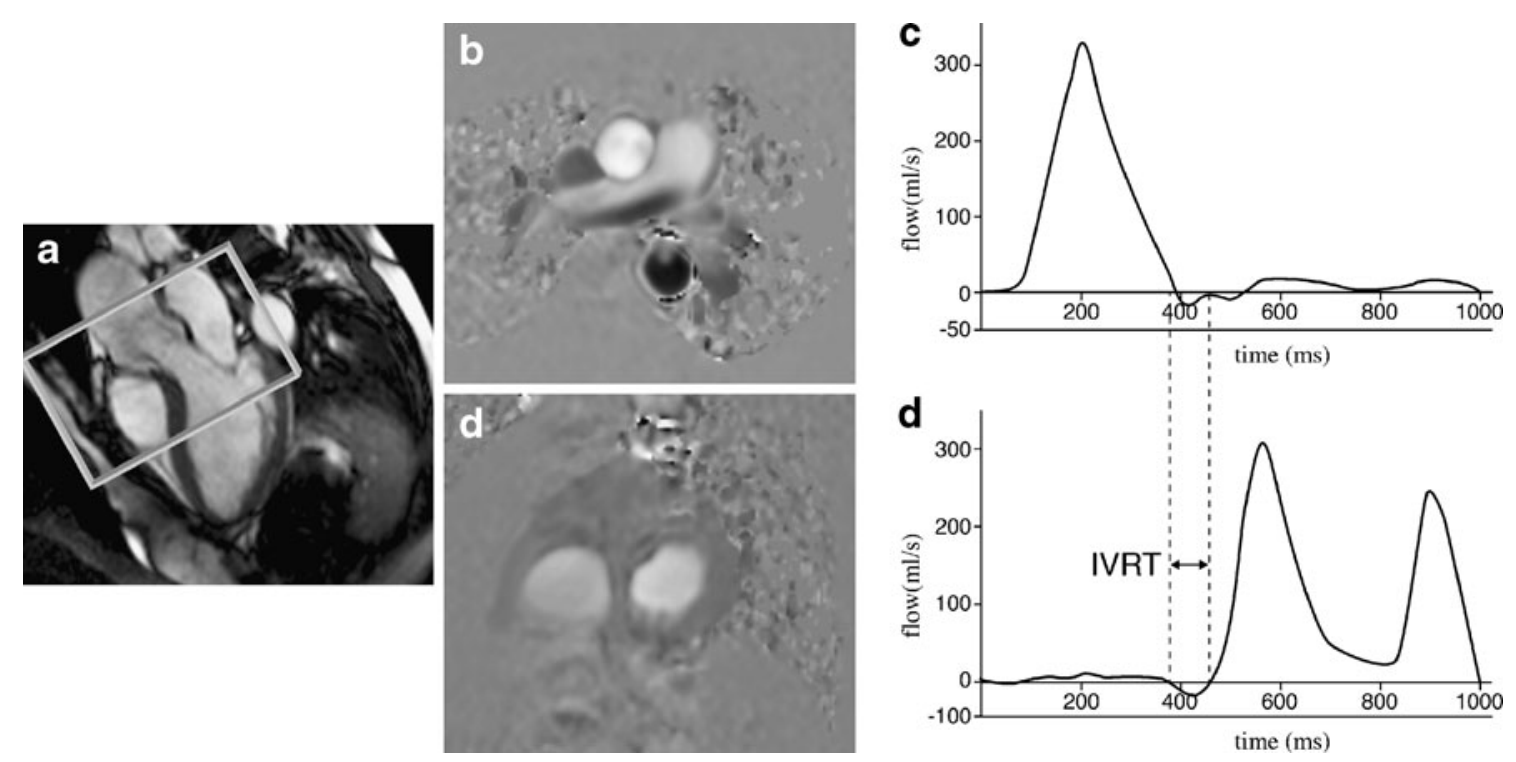

Fig. 4 Three-dimensional three-directional velocity-encoded MRI at the aorta and mitral valve (a) results in velocity images reconstructed at the ascending aorta (b) and the mitral valve (d). From the velocity images, time-flow rate graphs (c and e) are obtained from which the isovolumic relaxation time (IVRT) can be determined 
abnormality seen with diastolic dysfunction) [35]. A difference in $\mathrm{Ar}-\mathrm{A}$ duration $>30 \mathrm{~ms}$ indicates elevated LV end-diastolic pressure $[7 \bullet \bullet]$.

Only a few studies have shown the prognostic value of pulmonary venous flow indices, and practical limitations further minimize the role of these indices for diastolic function evaluation with echo Doppler. Atrial contraction may create wall motion artifacts obscuring the pulmonary flow velocity signal. Inconclusive results are found in cases of sinus tachycardia and first-degree atrioventricular block. With atrial fibrillation, loss of atrial contraction and relaxation leads to a reduction in pulmonary venous flow regardless of filling pressures. All these limitations may impede the use of pulmonary venous flow analysis for evaluation of diastolic function. Nevertheless, velocityencoded MRI offers a potentially useful alternative to pulsed-wave Doppler for pulmonary venous flow evaluation, similarly applied as for transmitral flow analysis. Both two-dimensional one-directional velocity-encoding at the pulmonary vein, as well as a three-dimensional threedirectional velocity-encoding approach of the intra-atrial blood flow field, may be followed in order to obtain the pulmonary venous time-velocity curve (Fig. 5) [36••]. The velocity sensitivity of the acquisition should be adjusted to a maximal velocity of $80 \mathrm{~cm} / \mathrm{s}$ to optimize the signal-tonoise ratio in these measurements. Similar to the transmitral velocity acquisition, the temporal resolution which determines the accuracy of wave form analysis is inferior for velocity-encoded MRI when compared to echo Doppler, and acquisition time is usually long. These may be drawbacks of the MRI approach.

\section{Flow Propagation Velocity}

Intraventricular flow is not only influenced by LV diastolic function but many variables may contribute to the appearance of the intraventricular blood flow field, such as viscoelastic properties, inertial force, LV geometry, systolic function, mitral valve function, and LV contractile function.
This makes it difficult to quantitatively relate intraventricular flow patterns to LV diastolic function. In case of normal LV function, the early filling wave propagates rapidly from mitral valve toward the apex, driven by a pressure gradient between atrium and apex [37]. This gradient creates the suction force which has been attributed to $\mathrm{LV}$ relaxation. In case of $\mathrm{HF}$, the pressure gradient decreases resulting in a slower propagation of the early filling wave. The flow propagation velocity $(\mathrm{Vp})$ can be measured with color M-mode Doppler from the mitral valve plane to $8 \mathrm{~cm}$ distally into the LV cavity [38]. $\mathrm{Vp}>$ $50 \mathrm{~cm} / \mathrm{s}$ is considered normal. In most patients with depressed $\mathrm{EF}, \mathrm{Vp}$ is reduced, with values of $\mathrm{E} / \mathrm{Vp}$ ratio $\geq$ 2.5 predicting elevated pulmonary capillary wedge pressures [39]. For patients with preserved EF, though, the E/ $\mathrm{Vp}$ ratio may be a less useful predictor of LV filling pressure since $\mathrm{Vp}$ can be misleadingly normal in these patients [39].

CMR in combination with three-dimensional threedirectional velocity-encoding may be a promising new application that can provide quantitative information on the intraventricular blood flow field (Fig. 6) [40], from which LV diastolic function indices readily can be assessed [27••]. Potentially, Vp may also be extracted from the time-resolved diastolic inflow velocity field. Currently, the long acquisition time $(>30 \mathrm{~min})$ to obtain this three-dimensional time-resolved intraventricular blood flow velocity field in the whole LV is the main drawback of this application.

\section{Myocardial Wall Motion and Strain}

Pulsed-wave tissue Doppler imaging (TDI) is a valuable echocardiographic technique that is routinely performed in an apical four-chamber view to evaluate diastolic function based on myocardial wall motion measurements. Mitral annular velocities in longitudinal direction are obtained at the intraventricular septum and lateral wall, and wave form analysis results in early $\mathrm{E}^{\prime}$ and late $\mathrm{A}^{\prime}$ annular velocities. $\mathrm{E}^{\prime}$
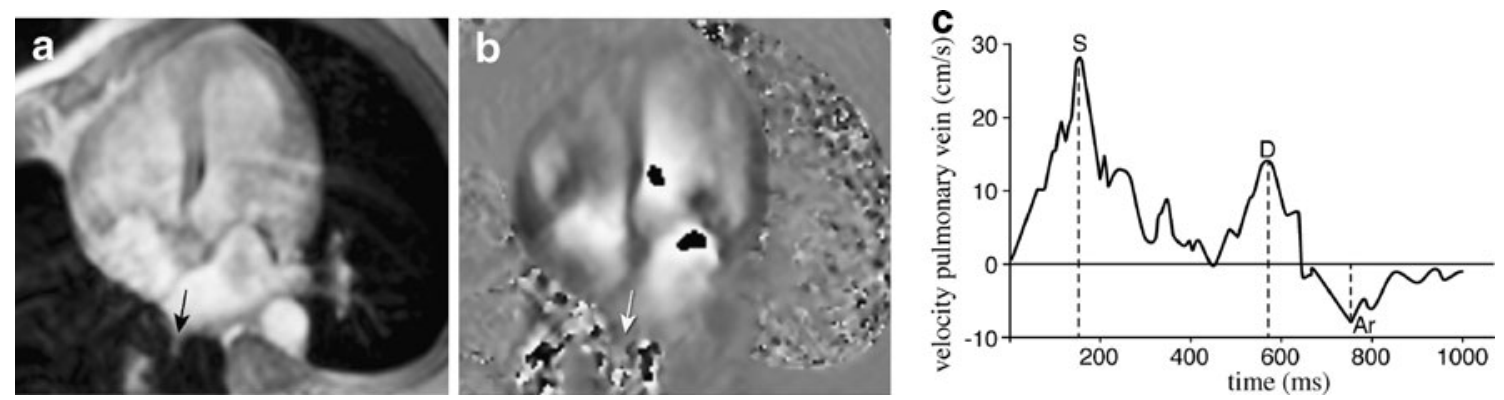

Fig. 5 In-plane one-directional velocity-encoded MRI in fourchamber orientation (magnitude image in a and velocity image in b). Velocity sampling at the pulmonary vein (arrow) results in a time- velocity graph (c), from which peak systolic velocity (S), peak anterograde diastolic velocity (D), and peak atrial reversal velocity (Ar) can be determined 


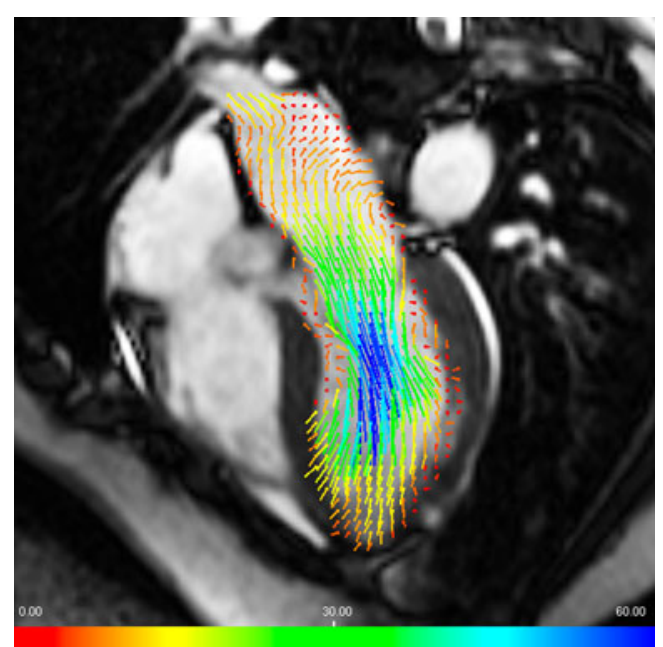

Fig. 6 The time-resolved velocity vector field (presented with color coding) on a four-chamber view is obtained from three-dimensional three-directional velocity-encoded MRI. From this inflow velocity vector field, the flow propagation velocity can be determined

is affected by LV relaxation, preload, systolic function, and LV minimal pressure $[7 \bullet \bullet]$. Therefore, $E^{\prime}$ and $E / E^{\prime}$ ratio play an important role in the estimation of LV filling pressures, but these indices should not be used as the sole data for diastolic function evaluation. The time difference $\left(\mathrm{T}_{\mathrm{E}-\mathrm{E}^{\prime}}\right)$ between onset of early peak filling velocity $\mathrm{E}$ and onset of $\mathrm{E}^{\prime}$ may provide supplemental information to $\mathrm{E} / \mathrm{E}^{\prime}$.

Septal $E^{\prime}$ is usually lower than lateral $E^{\prime}$; therefore, different cutoff values should be applied on the basis of $E^{\prime}$ sampling location. In the presence of regional dysfunction, the average of septal and lateral $\mathrm{E}^{\prime}$ is used [39]. In case of normal $L V$ function, septal $E^{\prime}>8 \mathrm{~cm} / \mathrm{s}$ and lateral $E^{\prime}>$ $10 \mathrm{~cm} / \mathrm{s}$. Using septal $\mathrm{E}^{\prime}$, a value of $\mathrm{E} / \mathrm{E}^{\prime}<8$ is usually associated with normal LV filling pressure, whereas $E / E^{\prime}>$ 15 is associated with increased filling pressure. Values between 8 and 15 remain inconclusive regarding LV filling pressures, suggesting usage of other echocardiographic indices for diastolic function evaluation. In such cases, $\mathrm{T}_{\mathrm{E}-\mathrm{E}^{\prime}}$ may be helpful, as IVRT/ $\mathrm{T}_{\mathrm{E}-\mathrm{E}^{\prime}}$ ratio $<2$ has reasonable accuracy in identifying patients with increased LV filling pressures [41].

There are a number of clinical settings described in which $\mathrm{E} / \mathrm{E}^{\prime}$ ratio and IVRT/ $\mathrm{T}_{\mathrm{E}-\mathrm{E}^{\prime}}$ ratio should not be used.
In normal subjects, $E^{\prime}$ is positively related to preload. Additionally, $\mathrm{E}^{\prime}$ is usually reduced in patients with mitral valve calcification or stenosis but increased in patients with mitral valve regurgitation. Septal $\mathrm{E}^{\prime}$ is usually increased in patients with constrictive pericarditis. Therefore, $\mathrm{E} / \mathrm{E}^{\prime}$ ratio and IVRT/ $\mathrm{T}_{\mathrm{E}-\mathrm{E}^{\prime}}$ ratio may be helpful in the evaluation of diastolic function, but specific patient characteristics need to be known.

Velocity-encoded MRI (Fig. 7) and TDI can be used interchangeably to assess longitudinal myocardial velocities [42]. This MRI approach has proven comparable value when applied for diastolic function assessment [43, 44•]. Drawbacks of this application are again the need of long scan time and the inferior temporal resolution when compared to TDI.

Two-dimensional and three-dimensional speckle-tracking echocardiography has recently emerged as a promising technique for evaluation of myocardial wall motion by strain analysis [45, 46], which was previously only possible by tissue tagging MRI. The deformation of the myocardium throughout the cardiac cycle can be analyzed by the mutual motion of acoustic speckles that are present inside the myocardium on the grayscale echocardiographic images. Assessment of radial and longitudinal strain (both representing LV expansion during mitral filling) as well as circumferential strain (representing the untwisting motion during isovolumic relaxation, representing the LV elastic recoil) may aid in identifying diastolic dysfunction. Clinical studies are required to establish the value of this new technique for diastolic function assessment.

Strain analysis from MRI tissue tagging, by single or complementary spatial modulation of magnetization (SPAMM or C-SPAMM) in combination with postprocessing approaches such as the automated harmonic phase (HARP) analysis [47], already is a well-established technique for identifying abnormal LV myocardial contraction and relaxation. In tissue tagging MRI, the myocardium is labeled by selective saturation pulses in planes perpendicular to the imaging plane, resulting in a grid-like pattern (Fig. 8) that can be tracked throughout the cardiac cycle, providing information on myocardial deformation, expressed in radial, longitudinal, and circumferential strain. This technique has not yet found widespread use in clinical
Fig. 7 One-directional velocityencoded MRI (a) of the longitudinal annular velocity in fourchamber orientation, sampled at the septum (arrow), results in time-velocity graph (b), from which early peak annular velocity $\left(E^{\prime}\right)$ can be determined
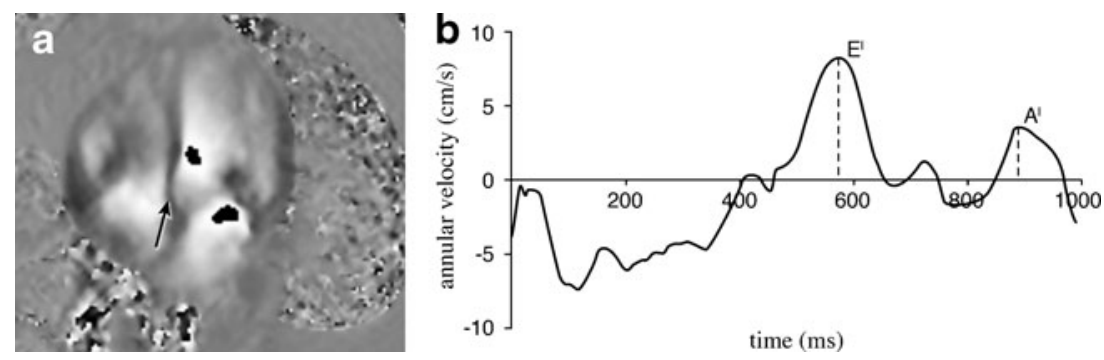


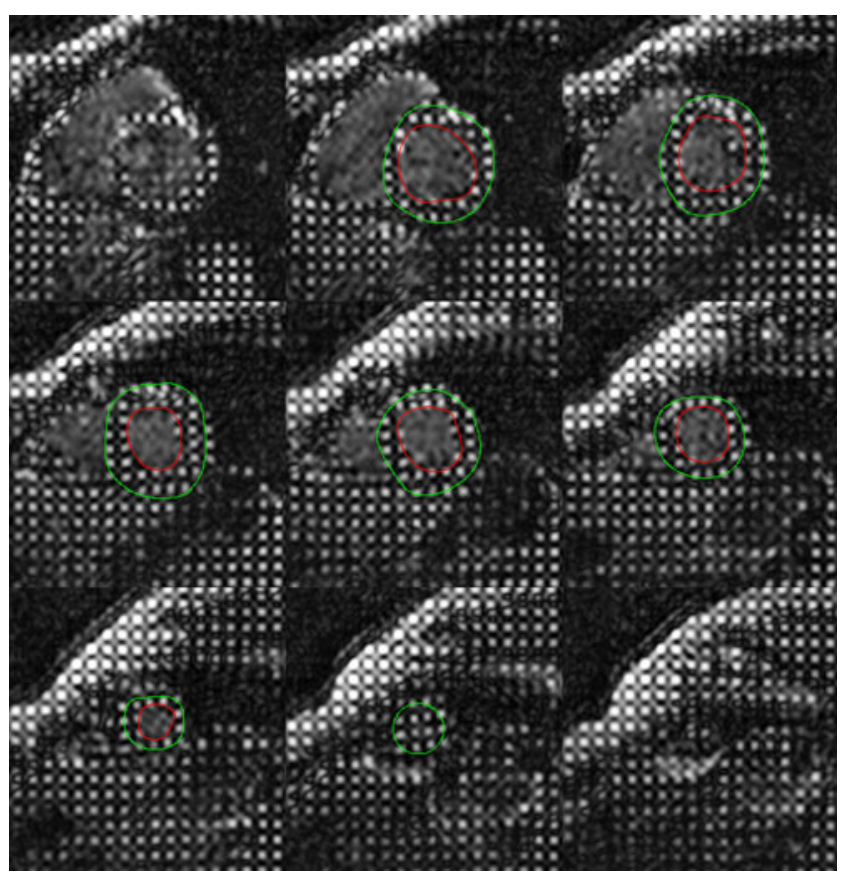

Fig. 8 MR tissue tagging in short-axis orientation for strain analysis of the myocardial motion

practice as several technical acquisition challenges still need to be overcome, such as improving the persistency of tag-lines throughout the cardiac cycle (persistency depends on T1 relaxation) as well as improving temporal and spatial resolution and accelerating scan time, as currently, the need for long breath-holding limits this application in $\mathrm{HF}$ patients. The value of improved acquisition techniques such as the application of displacement-encoded stimulated echoes (DENSE) [48] and strain-encoded imaging (SENC) [49] in diastolic function testing is still under investigation.

\section{Conclusions}

This review article focused on the role of CMR as alternative modality to echocardiography for the evaluation of LV diastolic function. CMR offers identification of LV and atrial geometry and function, intraventricular and pulmonary venous flow, as well as myocardial wall motion, which are all entities that are studied with echocardiography for diastolic function assessment (Table 2). CMR has already proven additional value, in some cases providing accuracy and reproducibility superior to echocardiography, and in other cases being complementary. General MRI contraindications (i.e., pacemakers, intracranial vascular clips, pregnancy, and claustrophobia) prohibit application of CMR for routine testing in all patients. Still, MRI is a patient-friendly and radiation-free imaging modality with three-dimensional quantification possibilities that makes no use of modeling assumptions and has no restrictions regarding the choice of imaging volume. Diastolic function testing with CMR can be integrated in a single comprehensive cardiac examination, e.g., combined with LV systolic function tests and localization and quantification of the extent of ischemic scar tissue and perfusion defects. New criteria for MRI-derived diastolic function indices still need to be determined through comparison studies as translation of the established echocardiographic cutoff values classifying diastolic dysfunction is not simply warranted.

Table 2 CMR for diastolic function testing

CMR acquisition Type of analysis Indices for diastolic function testing

1) Radial stack of long-axis or parallel LV time-volume relation from stack of short-axis covering whole LV

2) Parallel stack covering left atrium or 2-chamber and 4-chamber view

3) 1-directional or 3-directional VE MRI at the mitral valve

4) 1-directional or 3-directional VE MRI at the mitral and aortic valve

5) 1-directional or 3-directional VE MRI at the pulmonary veins

6) 3-directional VE MRI of the LV flow velocity field

7) 1-directional VE MRI of longitudinal annular velocity

8) MR tissue tagging in long-axis or short-axis orientation
$\mathrm{LV}$ time-volume relation from
planimetry

Left atrial size from planimetry or biplane Simpson's rule

Wave form analysis of mitral inflow pattern

Wave form analysis of mitral inflow and aortic outflow pattern

Wave form analysis of pulmonary venous flow pattern

Time-resolved flow velocity vector field during LV filling

Wave form analysis of myocardial motion pattern

Strain analysis
Early peak filling rate, time to peak filling rate, and atrial filling fraction

Left atrial size

Early peak filling rate (E) and atrial peak filling rate (A), E/A ratio, and deceleration time (DT) of early peak filling rate

Isovolumic relaxation time (IVRT)

Peak systolic velocity (S), peak anterograde diastolic velocity (D), S/ D ratio, systolic filling fraction, peak atrial reversal velocity (Ar), and duration between $\mathrm{Ar}$ and $\mathrm{A}$

Flow propagation velocity $(\mathrm{Vp})$ and $\mathrm{E} / \mathrm{Vp}$ ratio

Early peak annular velocity $\left(\mathrm{E}^{\prime}\right), \mathrm{E} / \mathrm{E}^{\prime}$ ratio, duration between $\mathrm{E}$ and $\mathrm{E}^{\prime}\left(\mathrm{T}_{\mathrm{E}-\mathrm{E}^{\prime}}\right)$, and IVRT/ $\mathrm{T}_{\mathrm{E}-\mathrm{E}^{\prime}}$ ratio

LV and atrial relaxation characteristics from longitudinal, circumferential, and radial strain

$L V$ left ventricle; $V E$ velocity-encoded. 
Acknowledgment Pieter van den Boogaard, Rob van der Geest, and Gerrit Kracht are gratefully acknowledged for acquisition and processing of the images used in the presented examples.

Disclosure No potential conflict of interest relevant to this article was reported.

Open Access This article is distributed under the terms of the Creative Commons Attribution Noncommercial License which permits any noncommercial use, distribution, and reproduction in any medium, provided the original author(s) and source are credited.

\section{References}

Papers of particular interest, published recently, have been highlighted as:

- Of importance

-. Of major imprtance

1. Owan TE, Hodge DO, Herges RM, et al. Trends in prevalence and outcome of heart failure with preserved ejection fraction. N Engl J Med. 2006;355:251-9.

2. Vasan RS, Larson MG, Benjamin EJ, et al. Congestive heart failure in subjects with normal versus reduced left ventricular ejection fraction: prevalence and mortality in a population-based cohort. J Am Coll Cardiol. 1999;33:1948-55.

3. $\mathrm{Yu} \mathrm{CM}$, Lin $\mathrm{H}$, Yang $\mathrm{H}$, et al. Progression of systolic abnormalities in patients with "isolated" diastolic heart failure and diastolic dysfunction. Circulation. 2002;105:1195-201.

4. Bursi F, Weston SA, Redfield MM, et al. Systolic and diastolic heart failure in the community. JAMA. 2006;296:2209-16.

5. Paulus WJ, Tschöpe C, Sanderson JE, et al. How to diagnose diastolic heart failure: a consensus statement on the diagnosis of heart failure with normal left ventricular ejection fraction by the Heart Failure and Echocardiography Associations of the European Society of Cardiology. Eur Heart J. 2007;28:2539-50.

6. Burkhoff D, Mirsky I, Suga H. Assessment of systolic and diastolic ventricular properties via pressure-volume analysis: a guide for clinical, translational, and basic researchers. Am J Physiol Heart Circ Physiol. 2005;289:H501-12.

7. •- Nagueh SF, Appleton CP, Gillebert TC, et al. Recommendations for the evaluation of left ventricular diastolic function by echocardiography. Eur J Echocardiogr. 2009; 10:165-193. Extensive overview of available echocardiographic techniques for diastolic function testing and recommended criteria for classifying the grade of dysfunction.

8. Attili AK, Schuster A, Nagel E, et al. Quantification in cardiac MRI: advances in image acquisition and processing. Int $\mathrm{J}$ Cardiovasc Imaging 2010; 26(Suppl 1):27-40. Review of recent advances in MRI acquisition techniques and post-processing methods for studying global and regional ventricular function, blood flow, perfusion, and scar.

9. Bellenger NG, Burgess MI, Ray SG, et al. Comparison of left ventricular ejection fraction and volumes in heart failure by echocardiography, radionuclide ventriculography and cardiovascular magnetic resonance; are they interchangeable? Eur Heart J. 2000;21:1387-96.

10. Thiele H, Nagel E, Paetsch I, et al. Functional cardiac MR imaging with steady-state free precession (SSFP) significantly improves endocardial border delineation without contrast agents. J Magn Reson Imaging. 2001;14:362-7.
11. Engels G, Müller E, Reynen K, et al. Evaluation of left ventricular inflow and volume by MR. Magn Reson Imaging. 1993;11:957-64.

12. Fujita N, Hartiala J, O'Sullivan M, et al. Assessment of left ventricular diastolic function in dilated cardiomyopathy with cine magnetic resonance imaging: effect of an angiotensin converting enzyme inhibitor, benazepril. Am Heart J. 1993;125:171-8.

13. Soldo SJ, Norris SL, Gober JR, et al. MRI-derived ventricular volume curves for the assessment of left ventricular function. Magn Reson Imaging. 1994;12:711-7.

14. Tsang TS, Barnes ME, Gersh BJ, et al. Left atrial volume as a morphophysiologic expression of left ventricular diastolic dysfunction and relation to cardiovascular risk burden. Am J Cardiol. 2002;90:1284-9.

15. Abhayaratna WP, Seward JB, Appleton CP, et al. Left atrial size: physiologic determinants and clinical applications. J Am Coll Cardiol. 2006;47:2357-63.

16. Westenberg JJ, van der Geest RJ, Lamb HJ, et al. MRI to evaluate left atrial and ventricular reverse remodeling after restrictive mitral annuloplasty in dilated cardiomyopathy. Circulation. 2005;112(9 Suppl):I437-42.

17. Sievers B, Kirchberg S, Addo M, et al. Assessment of left atrial volumes in sinus rhythm and atrial fibrillation using the biplane area-length method and cardiovascular magnetic resonance imaging with TrueFISP. J Cardiovasc Magn Reson. 2007;9:673-9.

18. Hudsmith LE, Petersen SE, Francis JM, et al. Normal human left and right ventricular and left atrial dimensions using steady state free precession magnetic resonance imaging. J Cardiovasc Magn Reson. 2005;7:775-82.

19. Appleton CP, Jensen JL, Hatle LK, Oh JK. Doppler evaluation of left and right ventricular diastolic function: a technical guide for obtaining optimal flow velocity recordings. J Am Soc Echocardiogr. 1997;10:271-92.

20. Rathi VK, Doyle M, Yamrozik J, et al. Routine evaluation of left ventricular diastolic function by cardiovascular magnetic resonance: a practical approach. J Cardiovasc Magn Reson. 2008;10:36.

21. Chai P, Mohiaddin R. How we perform cardiovascular magnetic resonance flow assessment using phase-contrast velocity mapping. J Cardiovasc Magn Reson. 2005;7:705-16.

22. Gatehouse PD, Keegan J, Crowe LA, et al. Applications of phasecontrast flow and velocity imaging in cardiovascular MRI. Eur Radiol. 2005;15:2172-84.

23. Paelinck BP, Lamb HJ, Bax JJ, et al. MR flow mapping of dobutamine-induced changes in diastolic heart function. J Magn Reson Imaging. 2004;19:176-81.

24. Kayser HW, Stoel BC, van der Wall EE, et al. MR velocity mapping of tricuspid flow: correction for through-plane motion. J Magn Reson Imaging. 1997;7:669-73.

25. Kozerke S, Schwitter J, Pedersen EM, et al. Aortic and mitral regurgitation: quantification using moving slice velocity mapping. J Magn Reson Imaging. 2001;14:106-12.

26. - Westenberg JJ, Roes SD, Ajmone Marsan N, et al. Mitral valve and tricuspid valve blood flow: accurate quantification with $3 \mathrm{D}$ velocity-encoded MR imaging with retrospective valve tracking. Radiology 2008, 249:792-800. This study shows improvement in transmitral flow assessment by applying three-dimensional threedirectional velocity-encoding.

27. •- Brandts A, Bertini M, van Dijk E, et al. Left ventricular diastolic function assessment from three-dimensional threedirectional velocity-encoded MRI with retrospective valve tracking. J Magn Reson Imaging, in press. This study compares conventional one-directional velocity-encoded MRI with threedirectional velocity-encoding for transmitral flow assessment and derived from this, diastolic function indices. The clinical value of these techniques for classifying diastolic dysfunction is compared with echocardiography. 
28. Roes SD, Hammer S, van der Geest RJ, et al. Flow assessment through four heart valves simultaneously using 3-dimensional 3directional velocity-encoded magnetic resonance imaging with retrospective valve tracking in healthy volunteers and patients with valvular regurgitation. Invest Radiol. 2009;44:669-75.

29. • Krishnamurthy R, Pednekar A, Cheong B, Muthupillai R. High temporal resolution SSFP cine MRI for estimation of left ventricular diastolic parameters. J Magn Reson Imaging 2010; 31:872-80. Use of high-end MRI scanners with modern hardware and accelerated scan techniques can establish high temporal resolution for cine imaging, improving accuracy for assessment of diastolic function indices.

30. Weiger M, Pruessmann KP, Boesiger P. Cardiac real-time imaging using SENSE. SENSitivity Encoding scheme. Magn Reson Med. 2000;43:177-84.

31. Tsao J, Kozerke S, Boesiger P, Pruessmann KP. Optimizing spatiotemporal sampling for k-t BLAST and k-t SENSE: application to high-resolution real-time cardiac steady-state free precession. Magn Reson Med. 2005;53:1372-82.

32. Smiseth OA, Thompson CR, Lohavanichbutr K, et al. The pulmonary venous systolic flow pulse - its origin and relationship to left atrial pressure. J Am Coll Cardiol. 1999;34:802-9.

33. Nishimura RA, Abel MD, Hatle LK, Tajik AJ. Relation of pulmonary vein to mitral flow velocities by transesophageal Doppler echocardiography. Effect of different loading conditions. Circulation. 1990;81:1488-97.

34. Keren G, Bier A, Sherez J, et al. Atrial contraction is an important determinant of pulmonary venous flow. J Am Coll Cardiol. 1986;7:693-5.

35. Klein AL, Tajik AJ. Doppler assessment of pulmonary venous flow in healthy subjects and in patients with heart disease. J Am Soc Echocardiogr. 1991;4:379-92.

36. • Rathi VK, Doyle M, Yamrozik J, et al. Routine evaluation of left ventricular diastolic function by cardiovascular magnetic resonance: a practical approach. J Cardiovasc Magn Reson. 2008; 10:36. Direct comparison between CMR and echocardiographic techniques for diastolic function testing.

37. Steine K, Stugaard M, Smiseth OA. Mechanisms of retarded apical filling in acute ischemic left ventricular failure. Circulation. 1999;99:2048-54.

38. Takatsuji H, Mikami T, Urasawa $\mathrm{K}$, et al. A new approach for evaluation of left ventricular diastolic function: spatial and temporal analysis of left ventricular filling flow propagation by color M-mode Doppler echocardiography. J Am Coll Cardiol. 1996;27:365-71.
39. Rivas-Gotz C, Manolios M, Thohan V, Nagueh SF. Impact of left ventricular ejection fraction on estimation of left ventricular filling pressures using tissue Doppler and flow propagation velocity. Am J Cardiol. 2003;91:780-4.

40. Bolger AF, Heiberg E, Karlsson M, et al. Transit of blood flow through the human left ventricle mapped by cardiovascular magnetic resonance. J Cardiovasc Magn Reson. 2007;9:741-7.

41. Rivas-Gotz C, Khoury DS, Manolios M, et al. Time interval between onset of mitral inflow and onset of early diastolic velocity by tissue Doppler: a novel index of left ventricular relaxation: experimental studies and clinical application. J Am Coll Cardiol. 2003;42:1463-70.

42. Westenberg JJ, Lamb HJ, van der Geest RJ, et al. Assessment of left ventricular dyssynchrony in patients with conduction delay and idiopathic dilated cardiomyopathy: head-to-head comparison between tissue Doppler imaging and velocityencoded magnetic resonance imaging. J Am Coll Cardiol. 2006;47:2042204-8

43. Paelinck BP, de Roos A, Bax JJ, et al. Feasibility of tissue magnetic resonance imaging: a pilot study in comparison with tissue Doppler imaging and invasive measurement. J Am Coll Cardiol. 2005;45:1109-16.

44. - Marsan NA, Westenberg JJ, Tops LF, et al. Comparison between tissue Doppler imaging and velocity-encoded magnetic resonance imaging for measurement of myocardial velocities, assessment of left ventricular dyssynchrony, and estimation of left ventricular filling pressures in patients with ischemic cardiomyopathy. Am J Cardiol. 2008; 102:1366-72. Direct comparison between tissue Doppler imaging and velocity-encoded MRI for assessment of diastolic function indices.

45. Wang J, Khoury DS, Yue Y, et al. Left ventricular untwisting rate by speckle tracking echocardiography. Circulation. 2007;116:2580-6.

46. Geyer H, Caracciolo G, Abe H, et al. Assessment of myocardial mechanics using speckle tracking echocardiography: fundamentals and clinical applications. J Am Soc Echocardiogr. 2010;23:351-69.

47. Osman NF, Kerwin WS, McVeigh ER, Prince JL. Cardiac motion tracking using CINE harmonic phase (HARP) magnetic resonance imaging. Magn Reson Med. 1999;42:1048-60.

48. Aletras AH, Ding S, Balaban RS, Wen H. DENSE: displacement encoding with stimulated echoes in cardiac functional MRI. J Magn Reson. 1999;137:247-52.

49. Korosoglou G, Youssef AA, Bilchick KC, et al. Real-time fast strain-encoded magnetic resonance imaging to evaluate regional myocardial function at 3.0 Tesla: comparison to conventional tagging. J Magn Reson Imaging. 2008;27:1012-8. 